(Bath: The Library, 1974-75).

2. Valentine De Bruin, "Sometimes Dirty Things Are Seen on the Screen," Journal of Academic Librarianship 3:256-66 (Nov. 1977).

3. Carolyn M. Cox and Bonnie Juergens, Microform Catalogs: A Viable Alternative for Texas Libraries (Dallas: AMIGOS Bibliographical Council, 1977). ERIC Document No. ED 149739.

4. James R. Dwyer, "Public Response to an Academic Library Microcatalog," Journal of Academic Librarianship 5:132-41 (July 1979).

5. Brett Butler, Martha W. West, and Brian Aveney, COM Catalog: Use and Evaluation: Report of a Field Study of the Los Angeles County Public Library System (rev. ed.; Los Altos: Information Access Corporation, 1979), 71p.

6. Theodora Hodges and Uri Bloch, "Fiche or Film for COM Catalogs-Two Use Tests" in Library Effectiveness: A State of the Art (Chicago: American Library Assn., 1980), p. $122-30$.

7. William Saffady, Computer-Output Microfilm: Its Library Applications (Chicago: American Library Assn., 1978), 190p.

8. Commercial COM Catalogs: How to Choose, When to Buy. Catalog Use Committee, Reference and Adult Services Division, American Library Association. (Chicago: American Library Assn., 1978), 47p.

9. DeBruin, "Dirty Things," p.266.

10. Hodges, "Fiche or Film," p.128.

11. Hodges to Crowley, September 1979.

\section{Electronic \\ Order Transmission}

James K. LONG: OCLC, Inc., Dublin, Ohio.

In this era of decreasing library allocation from the public sector, libraries are realizing increased benefits from the automation of the acquisitions process. The price of hardware is decreasing and the capabilities of the available offerings increasing. We have evolved from the small local library collection of data and printing of orders, through the book vendor offerings of an online connection to a single vendors inventory. These systems still required local mailing for all other vendor orders.
In 1981 we have seen a greater emphasis on electronic ordering. Memorial University in Canada has been experimenting in sending orders directly to John Coutts Library Services Ltd. in print format using the UTLAS CATSS System. Wayne State University is planning to use the Ringgold NONESUCH acquisitions system to transmit orders electronically to Book House using the BISAC tape format. Blackwell/ North America and the Academic Book Center have experimentally used WLN to receive test orders in a print file format. These all save time in getting the orders to the respective vendor. If sufficient volume can be generated there may be a savings in transmission costs over the U.S. Mail.

However, in order to realize maximum economics in this electronic process, four activities need to occur.

1. Acquisition orders must be collected from multiple libraries at a central site to generate volume for dispersal to multiple sites.

2. Standard formats need to be accepted and enforced for order transmission.

3. The ISBN must become a universally accepted part of the library acquisitions order.

4. The library must receive order status information from the vendor. Once again, this should occur via a standard data format.

At OCLC there were 113 libraries, as of November 1981, that could send printed orders from a central site to over 15,000 addresses of their choice. By July 1982 the projection is for over 200 libraries to be using the system. The library's order is batched by the vendor address that the library has specified. This process offers savings by sharing mail and printing costs between participants. With the proposed installation of direct transmission in 1982, this central collection will afford shared transmission costs. This is the type of centralized collection that maximizes the benefits of electronic ordering.

Within the book industry, standards for electronic data transmission for book ordering have been developed. In May of 1981 the Book Industry Systems Advisory Committee (BISAC), a subcommittee of the Book Industry Study Group (BISG), ap- 
proved the third version of their purchase order format. This is a simple format with fixed length fields and fixed length records. It was developed for tape transmission of book orders and relies heavily on the use of the International Standard Book Number (ISBN) for accuracy. ANSI Z39 subcommittee U is working on an ANSI purchase order data transmission format for libraries. This effort is in cooperation with BISAC. In 1981 there were nine book vendors using the BISAC purchase order format, including the large retail chains Walden and Dalton. There were also twelve vendors using the BISAC invoice format, five vendors using the title update format, and one vendor using the approved data transmission protocol (IBM 3780).

This book ordering activity and standards use is fine for the book vendors and retailers. But where are the libraries? OCLC plans to use BISAC data transmission protocol and fixed data format in their initial direct transmission effort. However, there are some real problems with these formats relative to library needs. First, the formats do not provide for serials ordering or renewal. Second, data fields in the format are fixed length. This is a real problem when ordering esoteric publications. Especially since the title and descriptor entries are a single field. Obviously there are many items that a library needs to order that cannot be supported by this current standard. OCLC and DataPhase have representatives on the BISAC purchase order subcommittee. This subcommittee is developing a variable length P.O. format. However, if there is to be real cooperation, and the accompanying economics, we must have more active participation from the library community.

The cataloger has the Library of Congress Catalog Number (LCCN). However, this is inadequate for library acquisitions. The ISBN was developed for acquisitions. The ISBN identifies the publishers or current distributor, the binding, etc., so necessary for accurate acquisitions. You can order music, maps, recordings, or film by using the ISBN. This is providing you order from a publisher that assigns ISBNs to those materials. It also assumes that you include the ISBN on the order. Baker and Taylor, Brodart, Random House, and McGraw-
Hill estimate less than 25 percent of their orders contain an ISBN. Yankee Book, Blackwell/North America, and the Book House report approximately 10 percent use on orders received. A significant number of these ISBNs are incorrect, obsolete, or otherwise erroneous. If we are to realize the tremendous economies possible with electronic transmission, we MUST have greater and more accurate use of the ISBN. It is simply uneconomical to transmit all of the data necessary to accurately identify a piece via the cataloging fields and subfields for every order; even if this information were available for ordering.

Another standard developed by BISAC is the Standard Address Number (SAN). All library vendors, public, academic, and school libraries have been assigned a SAN. Do you know yours? Do you know the SAN of your vendor? These SANs are available in your libraries' reference sections, as well as the online name-address directories that accompany the network acquisition systems. If electronic ordering is going to be used most effectively and economically, the SAN plays an important part. It is not economically efficient to transmit hundreds of characters of address information.

The last item that becomes feasible with electronic transmission is order status information. The day is gone when we can afford to keep thousands of dollars encumbered with acquisition pieces that are unavailable. The normal practice of automatic cancel after sixty or ninety days, keeps those monies committed. How much better would it be to know within twentyfour to forty-eight hours of an order that the material was unavailable. Those funds, that become more dear each year, could be recommitted to more available items. This would be advantageous to both the vendor, the library and ultimately the library patron. Both the BISAC invoice and title update formats have potential for use in reporting. It would be better, however, if we could derive a format specific for title status.

In closing, I urge you to use the ISBN and SAN; pursue avenues of collective ordering; and lastly, become active in the standards effort. It is the library that ultimately has the most to gain from a cooperative, coordi- 
nated, volume-oriented, resource-sharing electronic ordering process.

For information relative to BISAC transmission formats or BISAC membership, write to: Book Industry Systems Advisory Committee, 160 Fifth Ave., Suite 604, New York, NY 10010.

For input to BISAC purchase order formats, write to: J. K. Long, Chairman, BISAC P.O. Subcommittee, c/o OCLC, Inc., 6565 Frantz Rd., Dublin, OH 43017. (Mr. Long is also the library or network representative on the ISBN advisory council.)

For input to the ANSI Z39 P.O. transmission formats, write to: Mr. E. Muro, Chairman, Subcommittee U, c/o Baker \& Taylor Co., 6 Kirby Ave., Somerville, NJ 08876.

For problems with the ISBN and SAN, write to: Mr. Emory I Koltay, International Standard Book Numbering Agency, 1180 Avenue of the Americas, New York, NY 20036.

\section{Microcomputer Backup to Online Circulation}

\section{Sheila INTNER: Emory University, Atlanta, Georgia.}

Our primary objective in purchasing microcomputer systems for the Great Neck Library was to provide a better alternative to paper and pencil checkouts when our minicomputer-based CLSI LIBS 100 automated circulation system was down. Two difficult and lengthy downtime periods occurring shortly after going online convinced the administration that public service should not be jeopardized because of system failure. After investigation of the backup systems vended by Computer Translation, Inc. ${ }^{1}$ two of them were purchased in November 1980 .

Computer Translation, Inc. (CTI) sells a turnkey backup system based on an Apple II Plus microcomputer, with two mini-disk drives using $5 \frac{1}{4}$ " floppy diskettes, a TV monitor, and a switching system connecting the Apple to the LIBS 100 console and terminals. Software designed to interface with the CLSI system is part of the package. The backup collects and stores data for check-ins and checkouts and then dumps them into the database by simulating a terminal when the mini-main-frame is operational again. This requires dedicating a terminal to this process until complete. It can also be used alone as a portable unit for circulation purposes, or with any of the many Applesoft packages available, or with an Applesoft program of the user's own design.

Our initial experience in Great Neck was with a borrowed demonstration system, set up by a sympathetic CTI representative on the spur of the moment in tandem with and connected to the Main Library checkout station's CRT laser terminal after several days of downtime. The circulation staff cheered as the familiar prompts appeared on both screens. They used the CLSI equipment which they were accustomed to operating and the computer room staff learned to operate the CTI system. The ease with which the Apple could be transported to different locations in the building and the immediate relief it gave wherever it was connected, sometimes one checkout station, sometimes another, led us to put off deciding on a permanent installation at first. We thought it might be more advantageous to keep it on a rolling cart and use it wherever a terminal was down, or wherever the traffic appeared to be heaviest. We continued in this manner for a while even after both of our own Apple systems were delivered.

It soon became apparent that the Apple and its accompaniments, especially the switching system with its dangling cables, was a nuisance at the checkout counter. People with piles of books or records tended to nudge it dangerously close to the edge or jiggle its connections loose. The circulation staff didn't like waiting until someone from the computer room could be spared to bring up the system, secure the connections, and turn on the Apple. Also, although the Apple is a very reliable instrument which has given us negligible downtime, bumpy rides over various floors, carpets, lintels, and textured tiles occasionally loosened its chips and rendered it, too, inoperative.

CTI representatives were called in to make a more permanent installation for the Apple in our computer room, a simple operation requiring some additional cable. Se- 